\title{
Singular Continuous Spectrum under Rank One Perturbations and Localization for Random Hamiltonians
}

\author{
BARRY SIMON AND TOM WOLFF \\ California Institute of Technology
}

\begin{abstract}
We consider a selfadjoint operator, $A$, and a selfadjoint rank-one projection, $P$, onto a vector, $\varphi$, which is cyclic for $A$. In terms of the spectral measure $\mathrm{d} \mu_{\varphi}^{A}$, we give necessary and sufficient conditions for $A+\lambda P$ to have empty singular continuous spectrum or to have only point spectrum for a.e. $\lambda$. We apply these results to questions of localization in the one- and multi-dimensional Anderson models.
\end{abstract}

\section{Introduction}

In this note, we consider a situation already partially analyzed by Aronszajn [2] and Donoghue [13]. Let $A$ be a selfadjoint operator with simple spectrum on a Hilbert space, $\mathscr{H}$, and let $\varphi$ be a cyclic vector for $A$. Let $P$ be the projection $(\varphi, \cdot) \varphi$ and let $A_{\lambda}$ be the operator (selfadjoint on $D(A)$ )

$$
A_{\lambda}=A+\lambda P \text {. }
$$

By the spectral theorem, $\mathscr{H}$ is unitarily equivalent to $L^{2}\left(\mathbb{R}, d \mu_{0}\right)$ in such a way that $A$ is multiplication by $x$ and $\varphi \equiv 1$. Here $\mu_{0}$ is spectral measure of $\varphi$ for $A$. The idea of Aronszajn [2] is to relate spectral properties of $A_{\lambda}$ to $d \mu_{0}$, and in particular, to the Stieltjes transform

$$
F_{0}(z) \equiv \int \frac{d \mu_{0}(x)}{x-z}
$$

For example, one of the results of the Aronszajn-Donoghue analysis is that for any $\lambda \neq \lambda^{\prime}$ the spectral measures $d \mu_{\lambda}$ and $d \mu_{\lambda^{\prime}}$ (of $\varphi$ for $A_{\lambda}$ and $A_{\lambda^{\prime}}$, respectively) have singular parts which are mutually singular.

$F_{0}(z)$ is a Herglotz function, so the theory of boundary values of such functions (see [18], [24]) asserts that $F_{0}(x+i 0) \equiv \lim _{\varepsilon\llcorner 0} F_{0}(x+i \varepsilon)$ exists and is finite for a.e. real $x$. (The symbol a.e. without qualification will always mean with 
respect to Lebesgue measure.) We shall also need the function

$$
B(x) \equiv\left[\int(x-y)^{-2} d \mu_{0}(y)\right]^{-1}
$$

with the convention $\infty^{-1}=0$. Then we shall prove

THEOREM 1. The following are equivalent:

(a) For a.e. $\lambda, A_{\lambda}$ has empty singular continuous spectrum.

(b) For a.e. $x, B(x)+\mathscr{I}_{m} F_{0}(x+i 0)>0$.

Since $B(x)$ and $\mathscr{I}_{m} F_{0}(x+i 0)$ are both non-negative, statement (b) says that $\{x \mid B(x)>0\} \cup\left\{x \mid \mathscr{I}_{m} F_{0}(x+i 0)>0\right\}$ has full measure. In fact, since

$$
\mathscr{I}_{m} F_{0}(x+i \varepsilon)=\varepsilon \int\left[(x-y)^{2}+\varepsilon^{2}\right]^{-1} d \mu_{0}(y)<\varepsilon B(x)^{-1},
$$

these two sets are disjoint.

We shall also prove

THEOREM 2. The following are equivalent:

(a) For a.e. $\lambda, A_{\lambda}$ has only point spectrum.

(b) For a.e. $x, B(x)>0$.

While we started these theorems on all of $R$, they have local versions whose proofs are identical. For example:

THEOREM 2'. Fix an open interval $(a, b)$. The following are equivalent:

(a) For a.e. $\lambda, A_{\lambda}$ has only point spectrum in $(a, b)$.

(b) For a.e. $x$ in $(a, b), B(x)>0$.

These theorems will be a simple consequence of the ideas of AronszajnDonoghue and the fact (Theorem 5) that $d \eta(x) \equiv \int\left(1+\lambda^{2}\right)^{-1} d \mu_{\lambda}(x) d \lambda$ is mutually equivalent to Lebesgue measure. We discovered this later fact in trying to understand some work of Kotani [19] on the effect of boundary conditions on certain classes of random Hamiltonians. The analogue of Theorem 5, due to Carmona [5], played a major role in Kotani's work. In fact, this part of our work in a sense bears the same relation to Carmona-Kotani as Donoghue's work bears to that of Aronszajn.

While these two theorems are of some interest as abstract mathematics, their significance is increased by their connection to the theory of random Hamiltonians. In particular, we discuss the Anderson model here. One of us will discuss further applications elsewhere (see [20], [27]). The Anderson [1] model is 
an ensemble of operators on $l^{2}\left(Z^{\nu}\right)$,

$$
H_{\omega}=H_{0}+V_{\omega}
$$

where $H_{0}$ is a fixed operator

$$
\left(H_{0} u\right)(n)=\sum_{|j|=1} u(n+j)
$$

and $V_{\omega}$ is the random diagonal matrix

$$
\left(V_{\omega} u\right)(n)=V_{\omega}(n) u(n)
$$

and the $V .(n)$ are independent, identically distributed real random variables with distribution $d \kappa(x)$. It is a consequence of the ergodic theorem that the spectrum and spectral type is a.e. independent of $\omega$ (see [21]). Spectral properties of these operators have evoked considerable interest in both the physical and mathematical literature; see [6], [8], [29] for three recent reviews of the mathematical situation.

Under suitable conditions ( $\nu=1,2$ or when $\nu \geqq 3$, at energies near the edge of spec $\left(H_{\omega}\right)$ or when $V$ is "very random") it is believed that $H_{\omega}$ has only point spectrum dense in some regions; this has been proven in various circumstances (see below). This phenomenon is intimately related to the problem discussed in this paper. For, let $A_{\omega}=H_{\omega}-V_{\omega}(0) P$, where $P=\left(\delta_{0}, \cdot\right) \delta_{0}$ is the projection onto the vector $\delta_{0} \in l^{2}\left(\mathbb{Z}^{v}\right)$. Then

$$
H_{\omega}=A_{\omega}+\lambda P,
$$

where $\lambda$ is independent of $A_{\omega}$ and distributed according to the law $d \kappa$. Thus, if $d \kappa$ is absolutely continuous, Theorem 2 says that a sufficient condition for $H_{\omega}$ to have only point spectrum for a.e. $\omega$ is that $B_{\omega}(E)>0$ for a.e. $E$ (actually, we do not know that $\delta_{0}$ is cyclic for $A_{\omega}$, so $B_{\omega}(E)>0$ only implies that the spectral measure $d \mu_{\delta_{0}}^{\omega}$ is pure point-one then needs an additional argument; see Sections 5 and 6). And if $d \kappa$ has essential support $(-\infty, \infty)$, then $B_{\omega}(E)>0$ for a.e. $E$ is also necessary for $H_{\omega}$ to only have point spectrum.

Thus, Theorem 2 will allow a new proof of localization in the Anderson model. For $\nu=1$, we recover the result of Kunz-Souillard [21] and Delyon et al. [11]. Our proof is, we believe, more elementary than that in [11], [21] and requires much weaker hypotheses on $d \kappa$. It will even extend to fairly general situations where $V$ is no longer independent at distinct sites (see [20], [27]). Nevertheless, the translation invariance plays an important role in our proof but not in [11]. We emphasize that the method of Delyon et al. [11] can treat $H_{\omega}+V_{\omega}+W$ for arbitrary fixed $W$ (see [11]) and also the case of decaying randomness (see $[26],[12],[10])$, and we do not see how to discuss those cases with the method of this paper (the defect is not in Theorem 2, but in Furstenberg's theorem!). Our 
discussion of the $\nu=1$ case is closely related to a recent proof of Kotani [19] obtained independently of our work (we were motivated by a preliminary version of [19] which did not contain this result).

For $\nu>1$, we recover recent results of Frohlich et al. [15] and Goldsheid [16]. Since the appearance of our work is roughly simultaneous with theirs, we wish to emphasize that their work preceded ours by some months.

In Section 2, we prove Theorems 1 and 2. In Section 3, we present some simple examples that show that the results really do hold only for a.e. $\lambda$ and not all $\lambda$. In Section 4, we express the condition $B(E)>0$ in terms of the Green's function for $A$, i.e., matrix elements of the resolvent $\left(\delta_{0},(A-E+i \varepsilon)^{-1} \delta_{n}\right)$. In Section 5, we use Section 4 and work of Ishii [17] or Deift-Simon [9] to get localization in dimension 1. In Section 6, we use Section 4 and work of Fröhlich-Spencer [14] to get localization in dimension $\nu>1$. Finally, in Section 7, we discuss the connection of our work with that of Kotani [19]. In particular, we prove localization in $\nu>1$ dimension using only Theorem 5 and not Theorem 2 by exploiting Kotani's philosophy and ideas of Martinelli-Scoppola [22].

An announcement of our results appears in [28].

While we were proofreading the typescript of this manuscript, we received a paper from Delyon, Levy and Souillard [30] (with related works [31], [32] in preparation) related to our work here. Also motivated by Kotani, they prove localization in the multi-dimensional Anderson model from estimates of Fröhlich-Spencer [14]. Their proof is very close to the one we give in Section 7, the main difference being that in place of our abstract Theorem 5, they use an eigenvalue perturbation theory argument with roots in [21], [33].

We should like to thank S. Kotani for telling us of his work, and L. Arnold and W. Wischutz for organizing a conference which allowed one of us (B.S.) to learn of Kotani's work.

\section{Proof of the Main Theorems}

It is fairly easy to see that $\varphi$ is cyclic for $A_{\lambda}$, so to study the spectral properties of $A_{\lambda}$ we need only study the spectral measure $d \mu_{\lambda}$ of $\varphi$ for $A_{\lambda}$. One key element of the proof is the following result of Aronszajn [2]. We say that a measure $\eta$ is supported on $A$ if $\eta(\mathbb{R} \backslash A)=0$. $\mu_{\lambda}^{\text {a.c. }}, \mu_{\lambda}^{\text {s.c. }}, \mu_{\lambda}^{\text {p.p. }}$ denote the absolutely continuous, singular continuous and pure point parts of $\mu_{\lambda}$.

Theorem 3 (Aronszajn [2]). Let $X=\left\{x \mid \mathscr{I}_{m} F_{0}(x+i 0)>0\right\}, Y=\{x \mid B(x)$ $>0\}, Z=\mathbb{R} \backslash(X \cup Y)$. Then, for any $\lambda \neq 0, \mu_{\lambda}^{\text {a.c. }}$ is supported on $X, \mu_{\lambda}^{\text {p.p. }}$ is supported on $Y$, and $\mu_{\lambda}^{\text {s.c. }}$ is supported on $Z$.

Because we need some of the lemmas later and for the reader's convenience, we sketch the proof of this result. By definition of $d \mu_{\lambda}$,

$$
F_{\lambda}(z) \equiv \int \frac{d \mu_{\lambda}(x)}{x-z}=\left(\varphi,\left(A_{\lambda}-z\right)^{-1} \varphi\right)
$$


Taking expectation values of the second resolvent equation

$$
\left(A_{\lambda}-z\right)^{-1}=(A-z)^{-1}-\lambda(A-z)^{-1} P\left(A_{\lambda}-z\right)^{-1},
$$

we find that

$$
F_{\lambda}(z)=F_{0}(z)-\lambda F_{0}(z) F_{\lambda}(z)
$$

yielding Aronszajn's [2] fundamental relation:

$$
F_{\lambda}(z)=F_{0}(z) /\left(1+\lambda F_{0}(z)\right)
$$

From this, we first deduce the following variant of a result of Aronszajn [2].

THEOREM 4. Fix $\lambda \neq 0$. Then $d \mu_{\lambda}$ has a pure point at $x_{0} \in R$ if and only if

$$
\begin{aligned}
\lim _{\varepsilon \downarrow 0} F_{0}\left(x_{0}+i \varepsilon\right) & =-\lambda^{-1}, \\
B\left(x_{0}\right) & \neq 0
\end{aligned}
$$

Moreover, $\lambda^{-2} B\left(x_{0}\right)$ is precisely the $\mu_{\lambda}$ measure of $\left\{x_{0}\right\}$.

Proof: Since

$$
\begin{aligned}
& \mathscr{I}_{m} F_{\lambda}\left(x_{0}+i \varepsilon\right)=\varepsilon \int \frac{d \mu_{\lambda}(y)}{\left(x_{0}-y\right)^{2}+\varepsilon^{2}}, \\
& \mathscr{R}_{\varepsilon} F_{\lambda}\left(x_{0}+i \varepsilon\right)=\int \frac{\left(y-x_{0}\right) d \mu_{\lambda}(y)}{\left(y-x_{0}\right)^{2}+\varepsilon^{2}},
\end{aligned}
$$

the dominated convergence theorem implies that

$$
\mu_{\lambda}\left(\left\{x_{0}\right\}\right)=\lim _{\varepsilon \downarrow 0} \varepsilon \mathscr{I}_{m} F_{\lambda}\left(x_{0}+i \varepsilon\right)
$$

$$
\lim _{\varepsilon \downarrow 0} \varepsilon \mathscr{R} \varepsilon F_{\lambda}\left(x_{0}+i \varepsilon\right)=0
$$

Therefore, if $\mu_{\lambda}\left(\left\{x_{0}\right\}\right) \neq 0$, then $F_{\lambda}\left(x_{0}+i \varepsilon\right) \rightarrow \infty$ which, by (5b), implies that $F_{0}\left(x_{0}+i \varepsilon\right) \rightarrow-\lambda^{-1}$. 
Moreover, by the monotone convergence theorem, we always have

$$
\lim _{\varepsilon \downarrow 0} \varepsilon^{-1} \mathscr{I}_{m} F_{0}\left(x_{0}+i \varepsilon\right)=\lim _{\varepsilon \downarrow 0} \int \frac{d \mu_{0}(y)}{\left(x_{0}-y\right)^{2}+\varepsilon^{2}}=B\left(x_{0}\right)^{-1} \text {. }
$$

If (a) holds, then (6) implies that

$$
\lim _{\varepsilon \downarrow 0} F_{0} / \varepsilon F_{\lambda}=\lambda^{-1} i\left[\mu_{\lambda}\left(\left\{x_{0}\right\}\right)\right]^{-1},
$$

so

$$
\lim _{\varepsilon \downarrow 0} \mathscr{I}_{m}\left[F_{0} / \varepsilon F_{\lambda}\right]=\lambda^{-1} \mu_{\lambda}\left(\left\{x_{0}\right\}\right)^{-1}
$$

But, by (5),

$$
\mathscr{I}_{m}\left[F_{0} / \varepsilon F_{\lambda}\right]=\varepsilon^{-1} \mathscr{I}_{m}\left(1+\lambda F_{0}\right)=\varepsilon^{-1} \lambda \mathscr{I}_{m} F_{0}
$$

Relations (7)-(9) show that if (a) holds, then $\lambda^{2} \mu_{\lambda}\left(\left\{x_{0}\right\}\right)=B\left(x_{0}\right)$. Thus (a) and (b) imply that $d \mu_{\lambda}$ has a pure point at $x_{0}$, and conversely, if $d \mu_{\lambda}$ has an atom, we conclude first that (a) holds and then that (b) holds.

To complete the proof of Theorem 3, we need several facts:

(i) $d \mu_{\lambda}^{\text {a.c. }}=\pi^{-1} \mathscr{I}_{m} F_{\lambda}(x+i 0) d x$,

(ii) $d \mu_{\lambda}^{\text {s.c. }}$ is supported on $\left\{x \mid \overline{\lim } \mathscr{I}_{m} F_{\lambda}(x+i \varepsilon)=\infty\right)$,

(iii) $B(x)>0$ implies that $\lim _{\varepsilon \downarrow 0} F_{0}(x+i \varepsilon)$ exists and is real.

i) is a standard fact in the theory of Steiltjes transforms see [18], [24]). ii) is a weak form of the theorem of de Vallee Poussin [24] which gives the result with replaced by lim); we state it in this form, since this is easier to prove than the full theorem and suffices. iii) is a simple consequence of the dominated convergence theorem.

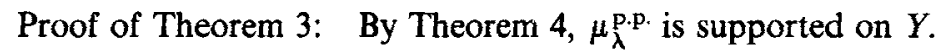

By ii) and iii), $d \mu_{\lambda}^{\text {s.c. is supported on }}$

$$
\{x \mid B(x)=0\} \cup\left\{x \mid B(x)>0, F_{0}(x+i 0)=-\lambda^{-1}\right\} .
$$

By Theorem 4, the second set is precisely the set of point masses for $\mu_{\lambda}$ and thus countable. Countable sets have $\mu^{\text {s.c. }}$ zero measure so we see that $\mu_{\lambda}^{\text {s.c. }}$ is supported on $\{x \mid B(x)=0\}$. If $\overline{\lim }_{\operatorname{m}} F_{\lambda}(x+i \varepsilon)=\infty$, then by (5), there must be a sequence $\varepsilon_{n}$ with $F_{0}\left(x+i \varepsilon_{n}\right) \rightarrow-\lambda^{-1}$ which is inconsistent with $I_{m} F_{0}(x+i 0)$ $>0$. Thus $\mu_{\lambda}^{\text {s.c. }}$ is supported on the complement of $X$; i.e., we have shown that $\mu_{\lambda}^{\text {s.c. }}$ is supported on $(\mathbb{R} \backslash X) \cap(\mathbb{R} \backslash Y)=\mathbb{R} \backslash(X \cup Y)$.

Finally, $\omega \mapsto \omega / 1+\lambda \omega$ is a transformation mapping $\left\{z \mid I_{m} z>0\right\}$ into itself with the inverse doing the same thing. Thus

$$
\left\{x \mid \mathscr{I}_{m} F d \lambda(x+i 0)>0\right\}=\left\{x \mid \mathscr{I}_{m} F_{0}(x+i 0)\right\}=X .
$$

By (i), we conclude that $X$ supports $d \mu_{\lambda}^{\text {a.c. }}$. 
The next result is an abstract analogue of a formula implicit in Carmona [5] and explicit in Kotani [19]. Since $\mu_{\lambda}$ is a probability measure for each $\lambda$ (if $\varphi$ is normalized), we can define a measure $\eta$ by

$$
\eta(A)=\int \mu_{\lambda}(A) \frac{d \lambda}{1+\lambda^{2}}
$$

for any set $A$.

THEOREM 5. $\eta$ is mutually equivalent to Lebesgue measure.

Proof: Since $|x-z|^{-1} \leqq\left|\mathscr{I}_{m} z\right|^{-1}$ for any real $x$, we see that when $\operatorname{Im} z>0$,

$$
H(z) \equiv \int \frac{d \eta(x)}{x-z}=\int \frac{d \lambda}{1+\lambda^{2}} F_{\lambda}(z) .
$$

Since an elementary contour integration shows that, for any $\omega$ with $\mathscr{I} m \omega>0$,

$$
\int \frac{d \lambda}{1+\lambda^{2}} \frac{\omega}{1+\lambda \omega}=\frac{\kappa}{\omega^{-1}-i}
$$

we conclude by (5) that

$$
H(z)=\kappa /\left(F_{0}(z)^{-1}-i\right) .
$$

Since $\mathscr{I}_{m} F_{0}>0$ in the upper half-plane, $|H(z)| \leqq \kappa$ and so $\mathscr{I}_{m} H(z) \leqq \kappa$. From this and the fact that $\kappa^{-1} \operatorname{Im} H(x+i \varepsilon) d x$ converges weakly to $d \eta$, it follows that

$$
d \eta \leqq d x
$$

so that $d \eta$ is absolutely continuous with respect to Lebesgue measure. On the other hand, taking the limit $\varepsilon \downarrow 0$ for $x_{0}+i \varepsilon$ in (11), we see that

$$
\left\{x \mid \mathscr{I}_{m} H(x+i 0)=0\right\}=\left\{x \mid F_{0}(x+i 0)=0\right\} .
$$

By general principles on the boundary values of analytic functions (see [18]), this last set has Lebesgue measure zero. Thus

$$
d \eta(x)=\kappa^{-1} \mathscr{J}_{m} H(x+i 0) d x
$$

is equivalent to Lebesgue measure. This result provides the essential link between sets of zero Lebesgue measure and sets of zero spectral measure, as follows.

Proof of Theorem 1: By Theorem 3, $\mu_{\lambda}^{\text {s.c. }}(\mathbb{R})=\mu_{\lambda}(\mathbb{R} \backslash(X \cup Y))$. Thus $A_{\lambda}$ has empty singular continuous spectrum for a.e. $\lambda$ if and only if $\mu_{\lambda}(\mathbb{R} \backslash(X \cup Y))$ 
is zero for a.e. $\lambda$. This happens if and only if

$$
\int d \lambda\left(1+\lambda^{2}\right)^{-1} \mu_{\lambda}(\mathbb{R} \backslash(X \cup Y))=0
$$

By Theorem 5, this holds if and only if $\mathbb{R} \backslash(X \cup Y)$ has Lebesgue measure zero, or equivalently if a.e. $x \in X \cup Y$.

Proof of Theorem 2: By Theorem 3, $\mu_{\lambda}^{\text {s.c. }}(\mathbb{R})+\mu_{\lambda}^{\text {a.c. }}(\mathbb{R})=\mu_{\lambda}(\mathbb{R} \backslash Y)$. Now, we argue as above.

\section{Some Examples}

Notice that a measure $d \mu_{0}$ determines both the operator $A$ and the vector $\varphi \equiv 1$, and so the entire example.

EXAmple 1. Let $\mu_{0}$ be the conventional Cantor measure. Any $x \in C$ has a base three expansion with only zeros and twos. For each $n,\{y \mid y \in C$ and $y$ and $x$ agree in their expansions for the first $n$ digits $\}$ are a distance at most $3^{-n}$ from $x$. Thus $\mu\left\{y \in C|| x-y \mid \leqq 3^{-n}\right\} \geqq 2^{-n}$ and so $\int d \mu(y) /|x-y|^{\alpha}=\infty$ if $\alpha \geqq$ $\log 2 / \log 3$. In particular, $B(x) \equiv 0$ on $C$. Thus, by Theorem $4, A_{\lambda}$ has eigenvalues only in the gaps of $C$. Since $|F(x+i 0)| \rightarrow \infty$ at edges of gaps, there is exactly one in each internal gap (for there is one solution of $F_{0}(x+i 0)=-\lambda^{-1}$ in each gap). Theorem 1 implies that for a.e. $\lambda, d \mu_{\lambda}$ has no singular continuous part. Actually, one can say more: if $x \in C$, then

$$
\mathscr{I}_{m} F_{0}\left(x+i 3^{-n}\right) \geqq \frac{1}{2} 3^{n} \mu\left\{y \in C|| x-y \mid \leqq 3^{-n}\right\} \rightarrow \infty
$$

so $I_{m} F_{\lambda}$ stays away from infinity. Thus, $d \mu_{\lambda \text {, sing }}$ is supported off $C=\sigma_{\text {ess }}\left(A_{\lambda}\right)$, i.e., $d \mu_{\lambda \text {, s.c. }}=0$. To summarize: If $\mu$ is the Cantor measure, for each $\lambda \neq 0, \mu_{\lambda}$ has only pure point spectrum; all eigenvalues are discrete, but the closure of the eigenvalues consists of all of $C . \mu_{\lambda}$ has thick point spectrum in the sense of [3]. Take $\tilde{A}=A+P$; we see that $\tilde{A}+\lambda P$ has singular continuous spectrum for exactly one value of $\lambda$. For the $\tilde{A}$ problem, Theorem 2 holds, but the conclusion is not for all $\lambda$.

EXAMPLE 2. Let $\delta_{x}$ be the unit mass at $x$. Let $\mu=\sum a_{n} d \mu_{n}$ with

$$
d \mu_{n}=\frac{1}{2^{n}} \sum_{j=1}^{2^{n}} \delta_{j / 2^{n}}
$$

Obviously we require $\sum a_{n}<\infty$. If $0 \leqq x \leqq 1$, (i.e. $x \in \operatorname{spec}(A)$ ), there is some $j$ 
with $\left|x-j / 2^{n}\right| \leqq 2^{-n}$ so

$$
\int|x-y|^{-2} d \mu_{n}(y) \geqq\left(2^{-n}\right)^{-2} 2^{-n}=2^{n}
$$

It follows that if $\sum 2^{n} a_{n}=\infty$, then $B(x)^{-1}=\infty$ on $[0,1]$, so by Theorem $4, \mu_{\lambda}$ has no pure points on $[0,1]$. Moreover, since $\left\{x \mid \mathscr{I}_{m} F_{0}(x+i 0)\right\}$ has measure zero, $d \mu_{\lambda}^{\text {a.c. }}=0$ for all $\lambda$. Thus $d \mu_{\text {sing }}$ has support $[0,1]$. There is one eigenvalue on $\mathbb{R} \backslash[0,1]$. To summarize, if $\sum a_{n}<\infty, \sum 2^{n} a_{n}=\infty$, then, when $\lambda \neq 0, \mu_{\lambda}$ has (except for one simple eigenvalue) only singular continuous spectrum $[0,1]$.

These two examples, closely related to examples in Aronszajn [2], present a striking contrast: A purely s.c. measure turning into pure point spectrum for all values of $\lambda$ and a pure point spectrum turning into (essentially) purely s.c. spectrum for all $\lambda$.

EXAMPLE 3. Let $x_{n}$ be arbitrary points. Let $0 \leqq a_{n} \leqq C \alpha^{n}$ with $\alpha<1$ and let $d \mu_{0}(x)=\sum a_{n} d_{x_{n}}$. We claim that $B(x)>0$ a.e. This follows from:

Proposition 6. If $0<\alpha<1$, then for a.e. $x$,

$$
H(x) \equiv \sum_{n} \alpha^{n}\left|x-x_{n}\right|^{-2}<\infty
$$

Proof: If $\left|x-x_{n}\right|>C^{-1 / 2} \alpha^{n / 4}$, then

$$
H(x)<\sum_{n} C \alpha^{n / 2}=C\left(1-\alpha^{1 / 2}\right)^{-1} .
$$

Thus

$$
\left|\left\{x \mid H(x) \geqq C\left(1-\alpha^{1 / 2}\right)^{-1}\right\}\right| \leqq \sum_{n} 2 C^{-1 / 2} \alpha^{n / 4}=2\left(1-\alpha^{1 / 4}\right)^{-1} C^{-1 / 2}
$$

goes to zero as $C \rightarrow \infty$.

Thus, in this case, $A_{\lambda}$ continues to have only pure point spectrum for a.e. $\lambda$.

Notice that if $A_{0}$ has eigenvalues at the points $j / 2^{n}, 0<j \leqq 2^{n}, n=1,2, \cdots$, then distinct choices of $\varphi$ correspond to distinct choices of weights $a_{n}$ in $d \mu_{0}(x)=\sum a_{n} \delta_{x_{n}}$. We can pick $\varphi$ to yield either Example 2 or 3 . This shows that, in general, different rank-one perturbations can yield dramatically different spectral consequences.

EXAMPLE 4. Let $d \mu_{0}=d \mu_{C}+d x \uparrow[0,1]$, where $d x$ is Lebesgue measure and $d \mu_{C}$ Cantor measure. Then $\mathscr{I}_{m} F(x+i 0)=1$ on $(0,1)$ so $A_{\lambda}$ has only a.c. spectrum on $[0,1]$ and one eigenvalue in $\mathbb{R} \backslash[0,1]$. The point of this example is that if $d \nu_{0}=d \mu_{\lambda_{0}}$, then $d \nu_{-\lambda_{0}}=d \mu_{0}$ has a singular continuous part. Thus, while 
$I_{m} F_{\lambda_{0}}(x+i 0)>0$ for a.e. $x$ in $[0,1]$, it can happen that $A \lambda^{\left(\nu_{0}\right)}$ has singular continuous spectrum for some $\lambda$ (although by Theorem 1 the set must have measure zero).

\section{A Criterion for Dense Point Spectrum in the Anderson Model}

We begin by writing an operator theoretic formula for $B(x)$. Let $\varphi \equiv \varphi_{0}$ and let $\left\{\varphi_{n}\right\}_{n \in I}$ be an orthonormal basis labeled by some index set $I$ including $\varphi_{0}$. Let

$$
G(n, m ; z) \equiv\left(\varphi_{n},(A-z)^{-1} \varphi_{m}\right)
$$

Then we have

Proposition 7. For $x \in R$,

$$
\int|x-y|^{-2} d \mu_{0}(y)=\lim _{\varepsilon \downarrow 0} \sum_{n \in I}|G(n, 0 ; x+i \varepsilon)|^{2}
$$

Proof: Clearly, by the monotone convergence theorem,

$$
\begin{aligned}
\int|x-y|^{-2} d \mu_{0}(y) & =\lim _{\varepsilon \downarrow 0} \int|x+i \varepsilon-y|^{-2} d \mu_{0}(y) \\
& =\lim _{\varepsilon \downarrow 0}\left(\varphi_{0},|A-x-i \varepsilon|^{-2} \varphi_{0}\right) \\
& =\lim _{\varepsilon \downarrow 0}\left(\varphi_{0},(A-x-i \varepsilon)^{-1}(A-x+i \varepsilon)^{-1} \varphi_{0}\right) \\
& =\lim _{\varepsilon \downarrow 0} \sum_{n \in I}|G(n, 0 ; x+i \varepsilon)|^{2} .
\end{aligned}
$$

Remark. As we have already noted, $B(x)>0$ supplies a one line proof (see equation (3)) that $\mathscr{I}_{m} F_{0}(x+i 0)=0$ and so $\mathscr{I}_{m} F_{\lambda}(x+i 0)=0$ for all $\lambda$. Thus the control of the Green's function in higher dimension by Fröhlich-Spencer [14] (see Section 6) immediately implies that $d \mu_{\lambda}^{\text {a.c. }}(x)=\kappa^{-1} \mathscr{I}_{m} F_{\lambda}(x+i 0) d x=0$, providing a quick proof that $d \mu^{\text {a.c. }}=0$ in that case. This gives a brief alternate to an argument of Martinelli-Scoppola [22].

For the remainder of this section, we specialize to discuss the Anderson model given by equation (4). We suppose $d \kappa$ obeys $\int\left(\log _{+}|x|\right) d \kappa<\infty$.

THEOREM 8. Consider the two statements for the $\nu$-dimensional Anderson model:

(a) For a.e. $\omega, H_{\omega}$ has only point spectrum in $(a, b)$.

(b) For a.e. $E \in(a, b)$ and a.e. $(\omega)$, 


$$
\lim _{\varepsilon \downarrow 0}\left[\sum_{n \in Z^{\nu}}|G(n, 0 ; E+i \varepsilon)|^{2}\right]<\infty .
$$

Then,

(i) if $d \kappa$ is purely a.c., (b) implies (a),

(ii) if $\nu=1$ and $d \kappa$ has a nonzero a.c. component, then (b) implies (a),

(iii) if the a.c. component of $d \kappa$ has essential support $(-\infty, \infty)($ e.g. $d \kappa$ is Gaussian), then (a) implies (b).

Proof: Theorems 1 and 2 are only stated for the cyclic case. They immediately apply to a general $A+\lambda P$ (with $P$ of rank 1 ) to the cyclic subspace generated by $A$ and $\operatorname{Ran} P$, and so they say something about the spectral measure $d \mu \tau$ associated to $\varphi \in \operatorname{Ran} P$.

If we fix $\omega$ but then vary $V(0)$ to a new value $\tilde{V}(0)$ holding $\{V(n)\}_{n \neq 0}$ fixed, we obtain the operators $A+\lambda P$ with $A=H_{\omega}, \lambda=\tilde{V}(0)-V_{\omega}(0), P=\left(\delta_{0}, \cdot\right) \delta_{0}$. Thus Theorem 2 and Proposition 7 say that $d \mu_{\omega ; \lambda}^{\delta_{0}}$ is pure point for a.e. $\omega, \lambda$ if and only if (12) holds for a.e. $E, \omega$. But by the independence of $V_{\omega}(0), d \mu_{\omega ;}^{\delta_{0}}$ with $\omega$ distributed by the i.i.d. process and $\lambda$ by $d \kappa\left(\cdot-V_{\omega}(0)\right)$ is precisely $d \mu_{\omega}^{\delta_{0}}$. Thus (iii) is immediate and (b) implies that $d \mu_{\omega}^{\delta_{0}}$ has only pure point spectrum for a.e. choice of $\{V(n)\}_{n \neq 0}$ and a.e. choice of $V(0)$ from the absolutely continuous component of $d \kappa$. Under hypothesis (i), this implies that $d \mu_{\omega}^{\delta_{0}}$ is pure point for a.e. $\omega$. By translation invariance, this is true for each $d \mu_{\omega}^{\delta_{n}}$ so $H_{\omega}$ has only pure point spectrum.

In case (ii), with positive probability $V(0)$ and $V(1)$ both lie in their absolutely continuous components. Thus, with positive probability, both $d \mu_{\omega}^{\delta_{0}}$ and $d \mu_{\omega}^{\delta_{2}}$ are pure point in $(a, b)$. Since $\delta_{0}, \delta_{1}$ are cyclic for $H_{\omega}, H_{\omega}$ has only pure point spectrum in $(a, b)$ with positive probability. But the spectral type of $H_{\omega}$ is a.e. constant (see [21]), so the positive probability result implies the result for a.e. $\omega$.

One can also deduce exponential decay of eigenfunctions from exponential decay of $G$ :

THEOREM 9. Suppose that $d \kappa$ is purely absolutely continuous, and that, for a.e. pairs $(\omega, E)\left(\omega \in \Omega\right.$, the probability space for $V^{(\omega)}$ and $E \in(a, b)$ with Lebesgue measure), we have that for $0<\varepsilon<1$

$$
|G(0, n ; E+i \varepsilon)| \leqq C_{\omega, E} \exp \{-a(E)|n|\} .
$$

Then, with probability 1 , the eigenfunctions $\varphi_{E}^{(\omega)}$ with $E \in(a, b)$ obey

$$
\left|\varphi_{E}^{(\omega)}(n)\right| \leqq D_{\omega, E} \exp \{-a(E)|n|\} .
$$

Proof: Taking matrix elements of (5a) for the pair $\left(\delta_{n}, \cdot \delta_{0}\right)$ we see that (with $\left.A_{\lambda}=H^{(\omega)}+\lambda \delta_{0}\right)$

$$
\left(\delta_{n},\left(A_{\lambda}-z\right)^{-1} \delta_{0}\right)=G(n, 0 ; z)\left(1-\lambda F_{\lambda}(z)\right) .
$$


If $E$ is an eigenvalue of $A_{\lambda}$,

$$
P_{\{E\}}=s-\lim (-i \varepsilon)\left(A_{\lambda}-x-i \varepsilon\right)^{-1}
$$

We conclude from (13) that

$$
\left|\left(\delta_{0}, P_{\{x\}} \delta_{n}\right)\right| \leqq C_{\omega, E} \exp \{-a(E)|n|\}\left(\delta_{0}, P_{\{x\}} \delta_{0}\right) .
$$

Since (13) implies (12), arguments similar to Theorem 8, using Theorem 5, show that, for a.e. pair ( $\omega, \lambda), H^{\omega}+\lambda \delta_{0}$ has eigenfunctions obeying (14). But since $d \kappa$ is purely a.c., this implies the result for a.e. $H^{\omega}$.

\section{Localization in the One-Dimensional Anderson Model}

THEOREM 10. The $\nu=1$ model with an arbitrary $d \kappa$ obeying

(a) dk has an a.c. component,

(b) $\int\left(\log _{+}|x|\right) d \kappa(x)<\infty$,

has only pure point spectrum.

Proof: By Theorem 8, we need only prove (12). By (b), $\gamma(E)$ exists, and by Furstenberg's theorem it is positive for all $E$. Thus, by Theorem 6.5 of Deift-Simon [9], for a.e. $(\omega, E), \int d \mu_{+}\left(E^{\prime}, \omega\right) /\left(E^{\prime}-E\right)^{2}<\infty$ or $E$ is an eigenvalue of $H_{\omega}^{+}$. Here $H_{\omega}^{+}$is the half-line operator with $u(0)=0$ boundary conditions and $d \mu_{+}$is the associated spectral measure for $\delta_{1}$. But the set of eigenvalues of $H_{\omega}^{+}$is countable, so, for a.e. $(\omega, E), \int d \mu_{+}\left(E^{\prime}, \omega\right) /\left(E^{\prime}-E\right)^{2}=S_{+}(\omega, E)<\infty$, a similar equation holding for $S_{-}(\omega, E)$. In terms of the $m_{ \pm}$functions of [25], [9], $S_{ \pm}(\omega, E)=\lim _{\varepsilon \downarrow 0} \varepsilon^{-1} \mathscr{I}_{m m_{ \pm}}(\omega, E+i \varepsilon)$ and

$$
S(\omega, E) \equiv \int \frac{d \mu_{\omega}\left(E^{\prime}\right)}{\left(E-E^{\prime}\right)^{2}}=\lim _{\varepsilon \downarrow 0} \varepsilon^{-1} \mathscr{I}_{m}\left(-\left[m_{+}+m_{-}+E+i \varepsilon-V_{\omega}(0)\right]^{-1}\right),
$$

where $d \mu_{\omega}$ is the spectral measure for $\delta_{0}$ and the operator $H_{\omega}$ on all of $Z$. Since $m_{+}+m_{-}+e-V_{\omega}(0)$ is a Herglotz function on the upper half-plane for each $\omega$, for a.e. pair $(\omega, E)$.

$$
\mathscr{I}_{m}\left(m_{+}(\omega, E+i 0)+m_{-}(\omega, E+i 0)+E-V_{\omega}(0)\right) \equiv \eta(\omega, E)
$$

is non-zero (see [18]). Thus

$$
S(\omega, E)=|\eta(\omega, E)|^{-2}\left\{s_{+}(\omega, E)+S_{-}(\omega, E)+1\right\}
$$

is a.e. finite and (12) holds.

Remarks 1. Ishii [17] has an argument which directly controls the Green's function and proves that (12) holds directly. His argument, while stated for 
bounded $V$ and the half-line, can be seen to only require $\int d \kappa(x)\left(\log _{+}(|x|)^{1+\delta}<\right.$ $\infty$, and to hold on the whole line.

2. A more direct proof of (12) exploiting the Osceledec theorem and Theorem 4 will be given in [27]. This applies also to the strip. Nonindependent $V_{\omega}(n)$ will also be discussed.

3. By the Osceledec theorem, one proves that eigenfunctions decay at the Lyaponov exponent rate, recovering a result of Carmona [4] and Craig-Simon [7].

\section{Localization in the Multi-Dimensional Anderson Model}

Several years ago, Fröhlich-Spencer [14] proved the following theorem:

TheOREM 11. Fix P. Let $\nu$ be general, and suppose that either:

(a) $d \kappa$ is absolutely continuous with $\|d \kappa / d E\|_{\infty}$ sufficiently small and $E$ is arbitrary, or

(b) $d \kappa$ is Gaussian and $|E|$ is large.

Then, for constants $C$ and $m$ depending only on $\|d \kappa / d E\|_{\infty}($ or $E)$ and $p$, one has

$$
\sup _{0<\varepsilon<1}\left|G_{\omega}(0, n ; E+i \varepsilon)\right| \leqq e^{m(N-|n|)}
$$

with probability at least $1-C N^{-p}$. Moreover, as $\|d \kappa / d E\|_{\infty}$ or $E^{-1}$ goes to zero, $m$ goes to infinity.

Since the set of $\omega$ where (15) holds is increasing with $N$ and the measure goes to zero, we see that, for a.e. $\omega$,

$$
\sup _{0<\varepsilon<1}\left|G_{\omega}(0 n ; E+i \varepsilon)\right| \leqq C e^{-m|n|}
$$

so that certainly (12) holds. Thus, using Theorems 8 and 9 , we recover the recent result of Fröhlich et al. [15] (a similar result has been announced by Goldsheid [16]):

TheOREM 12. Under the hypotheses of Theorem 11, $H$ has only dense point spectrum (for all $E$ if (a) holds and for $|E|$ large if (b) holds) with eigenfunctions decaying exponentially at a rate going to infinity as $\|d \kappa / d E\|_{\infty}$ or $|E|^{-1}$ goes to zero.

\section{Relation to Kotani's Work}

It seems to us that the proofs of localization presented herein should be thought of as occurring in two steps. We do not refer to the proof of Theorem 2 and the verification of $B(E)>0$, but rather to a different breakup of the 
analysis:

1. An argument that any singular continuous spectrum must lie in a set of Lebesgue measure zero of energy, a priori given by the potential outside some finite region $\Lambda$.

2. A proof that, for most choices of the potential inside $\Lambda$, any particular set of Lebesgue measure zero will have zero spectral measure.

From this point of view, the verification that $B(E)>0$ and Theorem 3 provide step 1 , while Theorem 5 is the key to step 2 . Thus, one understands the relation of this argument to the work of Kotani [19], which motivated parts of it. The two-step philosophy is implicit in Kotani, who uses ideas of Pastur [23] for step 1. Step 2 in his study of boundary condition variation is the argument of Carmona; in the Anderson model case, he uses an argument less general than Theorem 5.

One can obtain a partially alternate proof of Theorem 11 by using the analysis of Martinelli-Scoppola [22] for step 1. As in our argument, one uses Theorem 5 for step 2. Explicitly, following [22], one can use the Borel-Cantelli lemma and the estimates of Fröhlich-Spencer [14] to prove the following: Let $G_{n}(x, y ; E, \omega)$ be the resolvent of the finite matrix $H_{\omega, n}$ obtained by restricting $H_{\omega}$ to a box $\Lambda_{n}$ centered at 0 of side $l_{n}^{2} \rightarrow \infty\left(l_{n}=2^{n / 2}\right)$. Then, for each $E$ and a.e. $\omega$, there is an $n_{0}(\omega)$ such that

$$
\sup _{0<\varepsilon<1} G_{n}(x, y ; E+i \varepsilon, \omega) \leqq e^{-m|x-y|}
$$

if $n \geqq n_{0}(\omega), x, y \in \Lambda_{n},|x-y| \geqq l_{n}$.

Now let $\tilde{H}_{\omega}$ be $H_{\omega}+\lambda \delta_{0}$ for some $\lambda$, and suppose that $\psi$ is a polynomially bounded solution of

$$
\tilde{H}_{\omega} \psi=E \psi
$$

Then $\psi_{n} \equiv \psi$ restricted to $\Lambda_{n}$ obeys

$$
\left(H_{\omega}-E\right) \psi=\eta_{n}
$$

where $\eta_{n}$ is supported on $\{0\} \cup \partial \Lambda_{n}$ and $\left\|\eta_{n}\right\|_{\infty} \leqq C\left(1+\left|l_{n}^{p}\right|+|\lambda|\right)$. If $n \geqq n(\omega)$, $n \geqq 4$, and $2^{n-2} \leqq|x| \leqq 2^{n-1}$, we use (18) to obtain

$$
\psi(x)=\lim _{\varepsilon \downarrow 0} \sum_{y} G_{n}(x, y ; E+i \varepsilon, \omega) \eta_{n}(y) .
$$

For $y \in \operatorname{supp} \eta_{n}$ and $x$ as above, $|x-y| \geqq 2^{n-2} \geqq l_{n}$,

$$
|\psi(x)| \leqq C \exp \left\{-m 2^{n-2}\right\}\left(l_{n}^{p}+|\lambda|+1\right) l_{n}^{2 \nu-2},
$$

Thus $\psi$ decays exponentially. 
Essentially, we have made a very slight generalization of the MartinelliScoppola argument to show that for a.e. pairs $(E, \omega)$ any polynomially bounded solution of (17) decays exponentially. Given the philosophy and Theorem 5, one obtains the promised alternative proof of Theorem 11 .

Acknowledgment. The research of the first author was partially supported by USNSF under Grants MCS-81-20833 and that of the second by DMS-84-07099.

\section{Bibliography}

[1] Anderson, P. W., Absence of diffusion in certain random lattices, Phys. Rev. 109, 1958, p. 1492.

[2] Aronszajn, N., On a problem of Weyl in the theory of singular Sturm-Liouville equations, Am. J. Math. 79, 1957, pp. 597-610.

[3] Avron, J., and Simon, B., Transient and recurrent spectrum, J. Func. Anal. 43, 1981, pp. 1-31.

[4] Carmona, R., Exponential localization in one-dimensional disordered systems, Duke Math. J. 49, 1982, p. 191.

[5] Carmona, R., One-dimensional Schrödinger operators with random or deterministic potentials: New spectral types, J. Func. Anal. 51, 1983, pp. 229-258.

[6] Carmona, R., Lectures on Random Schrödinger Operators, 14th St. Flour Prob. Summer School Lectures.

[7] Craig, W., and Simon, B., Subharmonicity of the Lyaponov index, Duke Math. J. 50, 1983, pp. 551-560.

[8] Cycon, H., Froese, R., Kirsch, W., and Simon, B., Topics in the Theory of Schrödinger Operators, Springer, to appear.

[9] Deift, P., and Simon, B., Almost periodic Schrödinger operators, III. The absolutely continuous spectrum in one dimension, Comm. Math. Phys. 90, 1983, pp. 389-411.

[10] Delyon, F., Apparition of purely singular continuous spectrum in a class of random Schrödinger operators J. Stat. Phys., to appear.

[11] Delyon, F., Kunz, H., and Souillard, B., One-dimensional wave equations in disordered media, J. Phys. A16, 1983, p. 25.

[12] Delyon, F., Simon, B., and Souillard, B., From power pure point to continuous spectrum in disordered systems, Ann. Inst. H. Poincaré, 42, 1985, p. 283.

[13] Donoghue, W., On the perturbation of spectra, Comm. Pure Appl. Math. Vol. 18, 1965, pp. 559-579.

[14] Fröhlich, J., and Spencer, T., Absence of diffusion in the Anderson tight binding model for large disorder or low energy, Comm. Math. Phys. 88, 1983, pp. 151-189.

[15] Fröhlich, J., Martinelli, F., Scoppola, E, and Spencer, T., Anderson localization for large disorder or low energy, Rome, preprint.

[16] Goldsheid, I., Talk at Tashkent Conference on Information Theory, Sept., 1984.

[17] Ishii, K., Localization of eigenstates and transport phenomena in one-dimensional disordered systems, Supp. Prog. Theor. Phys. 53, 1973, pp. 77.

[18] Katznelson, Y., An Introduction to Harmonic Analysis, Dover, 1976.

[19] Kotani, S., Lyaponov exponents and spectra for one-dimensional random Schrödinger operators, to appear in Proc. 1984 AMS Conference on "Random Matrices and their Applications"; Lyaponov exponents and point spectrum for one-dimensional random Schrödinger operators, in preparation.

[20] Kotani, S., and Simon, B., Localization in general one-dimensional random systems, II. Continuum Schrödinger operators, in preparation.

[21] Kunz, H., and Souillard, B., Sur le spectre des operateurs aux differences finies aleatoires, Comm. Math. Phys. 78, 1980, pp. 201-246. 
[22] Martinelli, F., and Scoppola, E., A remark on the absence of absolutely continuous spectrum in the Anderson model for large disorder or low energy, Comm. Math. Phys., 97, 1985, p. 465.

[23] Pastur, L., Spectral properties of disordered systems in one-body approximation, Comm. Math. Phys. 75, 1980, p. 179.

[24] Saks, S., Theory of the Integral, G. E. Stechert, New York, 1937.

[25] Simon, B., Kotani theory for one-dimensional stochastic Jacobi matrices, Comm. Math. Phys. 89, 1983 , p. 227.

[26] Simon, B., Some Jacobi matrices with decaying potential and dense point spectrum, Comm. Math. Phys. 87, 1982, pp. 253-258.

[27] Simon, B., Localization in general one-dimensional random systems, I. Jacobi matrices, Comm. Math. Phys., to appear.

[28] Simon, B., Taylor, M., and Wolff, T., Some rigorous results for the Anderson model, submitted to Phys. Rev. Lett.

[29] Spencer, T., The Schrödinger equation with a random potential, a mathematical review; to appear in Proc., 1984, Les Houches Summer School.

[30] Delyon, F., Levy, Y., and Souillard, B., Anderson localization for muiti-dimensionai systems at large disorder or large energy, Comm. Math. Phys., to appear.

[31] Delyon, F., Levy, Y., and Souillard, B., Anderson localization for one and quasi one-dimensional systems, in preparation.

[32] Delyon, F., Levy, Y., and Souillard, B., An approach "a la Borland" to Anderson localization in multi-dimensional disordered systems, Phys. Rev. Lett, 55, 1985, p. 618.

[33] Wegner, F., Bounded on the density of states of disordered systems, Z. Phys. B44, 1981, pp. 9-15.

\section{Received March, 1985}

